\title{
Painel MDP com resina poliuretana à base de óleo de mamona com adição de cimento
}

\author{
Medium density particleboard made with castor oil-based \\ polyurethane and with cement addition
}

\begin{abstract}
João Paulo Monteiro Couto
Maria Fátima Nascimento

Vinícius Borges de Moura Aquino

Tulio Hallak Panzera

André Luis Christoforo

Francisco Antonio Rocco Lahr

\section{Resumo}

$\mathbf{N}$

este trabalho foi investigada a influência da inclusão de frações mássicas de cimento Portland (CP-V ARI) [0\%, 10\%, 20\% e 30\%] a título de simular parcelas de resíduo de construção civil que tenha frações cimentícias não hidratadas na produção de painéis de partículas compostos de madeira de Pinus sp. e resina poliuretana à base de mamona, buscando avaliar o potencial de painéis produzidos com adição de materiais granulares como resíduos de construção civil. A caracterização física e mecânica de painéis MDP com adição de cimento foi feita com o auxílio da norma brasileira NBR 14810 e da análise de variância (ANOVA), sendo analisadas as propriedades físicas e mecânicas. Foram fabricados 4 painéis de $400 \mathrm{~mm}$ x 400 mm x $10 \mathrm{~mm}$ com partículas de Pinus sp. e resina poliuretana à base de óleo de mamona com teor de adesivo igual a 10\% em relação à massa seca de partículas, para cada tratamento, totalizando 16 painéis. Para cada painel foram retirados 3 corpos de prova $(\mathrm{CP})$ para avaliação das propriedades físicas e mecânicas. A adição de frações mássicas de cimento acima de $10 \%$ impactou negativamente as propriedades mecânicas dos painéis, por ter diminuído os valores de MOE e MOR As propriedades de densidade, absorção e inchamento se mantiveram dentro dos requisitos da norma.
\end{abstract}

\footnotetext{
1'João Paulo Monteiro Couto 1 Universidade Federal de São Carlos São Carlos - SP - Brasil

${ }^{2}$ Maria Fátima Nascimento ${ }^{2}$ Universidade de São Paulo São Carlos - SP - Brasil

${ }^{3}$ Vinícius Borges de Moura Aquino

${ }^{3}$ Universidade Federal do Sul e Sudeste do Pará

Santana do Araguaia - PA - Brasil
}

${ }^{4}$ Tulio HallakPanzera ${ }^{4}$ Universidade Federal de São João Del-Rei

São João Del Rei - MG - Brasil

${ }^{5}$ André LuisChristoforo ${ }^{5}$ Universidade Federal de São Carlos São Carlos - SP - Brasil

${ }^{6}$ Francisco Antonio Rocco Lahr 'Universidade de São Paulo São Carlos - SP - Brasil

Recebido em 27/05/19 Aceito em 15/11/19
Palavras-chave: Painéis de partículas de média densidade (MDP). Pinus. Cimento. Análise de variância (ANOVA).

\section{Abstract}

This research study investigates the influence of the inclusion of Portland cement mass fractions (CP-V ARI) [0\%,10\%, 20\% and 30\%] on the production of composite wood panels made with Pinus sp. and castor oil-based polyurethane resin, with the aim of evaluating the potential of panels produced with the addition of granular materials as construction waste. The characterisation was performed with the aid of the Brazilian standard NBR 14810 and an analysis of variance (ANOVA), analysing the physical and mechanical properties. Four $400 \mathrm{~mm} \times 400$ mm $\times 10 \mathrm{~mm}$ panels were manufactured for each treatment, totalling 16 panels. For each panel, 3 test specimens (CP) were taken for an evaluation oftheir physical and mechanical properties. The addition of mass fractions of cement larger than $10 \%$ are detrimental to the mechanical properties of the panels, reducing the MOE and MOR values. The density, absorption and swelling properties remained within the requirements of the standard.

Keywords: Medium density particleboard. Pinus. Cement. Analysis of variance (ANOVA)

COUTO, J. P. M.; NASCIMENTO, M. F.; AQUINO, V. B. de M.; PANZERA, T. H.; CHRISTOFORO, A. L.; LAHR, F. A. R. 


\section{Introdução}

O Brasil, sendo historicamente ligado à tradição florestal e com ampla cobertura florestal, vem buscando diferentes maneiras de emprego da madeira em sua economia (ARAUJO et al., 2018; BEECH et al., 2017; MOLINA; CALIL JUNIOR, 2010; NASCIMENTO et al., 2015; SOTSEK; SANTOS, 2018; STEEGE et al., 2016). Entre as várias formas de se aumentar a racionalização dos produtos à base de madeira, destacam-se os produtos engenheirados à base de madeira, como os painéis MDP (medium density particleboard), os painéis MDF (medium density fiberboard) e os painéis de lascas de madeira (oriented strand board, OSB) (SEMPLE; ZHANG; SMITH, 2015; SILVA et al., 2016; SOUZA et al., 2014; THOMPSON et al., 2002).

Esses painéis de madeira constituem hoje o terceiro maior segmento industrial de base florestal no país, ficando atrás apenas da indústria do papel e da siderurgia (INDÚSTRIA..., 2017). Essa posição significativa é resultado das diversas vantagens proporcionadas pelos painéis engenheirados à base de madeira, como menor produção de resíduos em sua produção, possibilidade de reutilização de madeiras provenientes do setor primário de processamento do material e maior uniformidade de propriedades se comparadas com as da madeira serrada (CHOI et al., 2018; DERKOWSKI; MIRSKI; MAJKA, 2015; RAMAGE et al., 2017; SOUZA et al., 2018). Além disso, seu papel na indústria moveleira e da construção civil vem se tornando cada vez mais significativo (GONÇALEZ et al., 2006; MANTANIS et al., 2018).

$\mathrm{O}$ painel engenheirado à base de madeira pode ser definido como sendo formado por partículas de madeira de variada dimensão, impregnadas de resinas sintéticas e naturais, sob a ação de pressão e calor. Entre as variáveis a serem consideradas na produção dos painéis, são considerados o teor de umidade, a temperatura, a resistência mecânica e a aplicabilidade (GARZÓN-BARRERO et al., 2016; IHNÁT et al., 2017; SILVA et al., 2018, 2014; ZAU et al., 2014).

Na produção de painéis MDP utiliza-se madeira advinda de florestas plantadas e reflorestadas, destacando-se o gênero Eucalyptus e mais especificamente a espécie Pinus, devido a seu crescimento mais rápido se comparado a outras espécies (IWAKIRI et al., 2015; MACEDO et al., 2015; NEGRÃO et al., 2014).

No Brasil, os ensaios dos painéis de partículas de madeira a fim de obter resultados de características físicas e mecânicas seguem as premissas e os métodos de cálculo do documento normativo NBR 14810 (ABNT, 2013). Essa norma também apresenta os valores mínimos e/ou máximos (requisitos) das características obtidas pelos ensaios que os painéis devem apresentar.

Um dos adesivos mais utilizados na fabricação dos painéis de partículas é a ureia-formaldeído, que apresenta boa resistência à umidade, baixo custo, cura à temperatura ambiente ou alta temperatura $\left(130{ }^{\circ} \mathrm{C}\right.$ a $\left.200{ }^{\circ} \mathrm{C}\right)$ (MANTANIS et al., 2018; OLIVEIRA et al., 2017; ROFFAEL; SCHNEIDER; DIX, 2015). Entretanto, esse adesivo emite gases tóxicos, não é renovável e dificulta a reciclagem dos produtos finais. Um adesivo menos utilizado, mas amplamente encontrado em pesquisas, é a resina obtida a partir do óleo de mamona (resina poliuretana à base de mamona), um produto de origem da biomassa que apresenta temperatura de prensagem (a partir de $60{ }^{\circ} \mathrm{C}$ ) significativamente menor que a do ureia-formaldeído para a fabricação dos painéis (BERTOLINI et al., 2014a; CARVALHO et al., 2014; SOUZA et al., 2018). Além disso, é comum a utilização de preservantes hidrossolúveis como o arseniato de cobre cromatado (CCA), que confere proteção contra fungos, insetos e brocas marinhas, não exalando odores e vapores tóxicos ao homem, nem aumentando a combustibilidade do painel (ALMEIDA et al., 2019; BERTOLINI et al., 2014b; FERRO et al., 2016).

Diversos estudos vêm sendo desenvolvidos com o intuito de analisar o potencial de painéis MDP com adição de outros materiais, como compósitos laminados como fibras de vidro, carbono, sisal e piaçava, serragem e cimento em matriz epóxi, e resíduos agrícolas como o pergaminho de café (BERTOLINI et al., 2014a; GARZÓN-BARRERO et al., 2016; SCATOLINO et al., 2017; SOUZA et al., 2014; VALARELLI et al., 2014). Porém, não se observam na literatura estudos de painéis MDP feitos com resina poliuretana à base de óleo de mamona, partículas de madeira tratadas com preservativo CCA e outros materiais de construção, como cimento, e resíduos de demolição e construção. Dessa forma, há a necessidade de estudos sobre outros tipos de materiais, como o cimento e materiais agregados provenientes de resíduos de construção civil.

O cimento é um material muito utilizado na construção civil, tendo baixo custo ante outros materiais da construção, bom controle granulométrico e elevada resistência mecânica à compressão, superior à da madeira (AMIANDAMHEN; MEINCKEN; TYHODA, 2016; LI; SHI, 2019; MISSAGIA et al., 2013; PANZERA et al., 2010). A facilidade de acesso a esse material, a possibilidade de notar hidratação não desejada rapidamente e o fato de que partículas de resíduos de construção e demolição com diâmetros

662 Couto, J. P. M.; Nascimento, M. F.; Aquino, V. B. de M.; Panzera, T. H.; Christoforo, A. L.; Lahr, F. A. R. 
menores que $0,15 \mathrm{~mm}$ possuem maior possibilidade de ter em sua composição partículas não hidratadas de cimento (MONTGOMERY, 1998) tornam o cimento Portland CPV-ARI uma alternativa para uma análise do comportamento de materiais agregados na produção de painéis de partículas de média densidade. Como na produção dos painéis MDP com resina poliuretana não há adição de água (FERRO et al., 2019; SILVA et al., 2015), o cimento tem função de preenchimento de vazios. Esse aspecto deve ser tratado em futuras pesquisas, que analisem o uso de resíduo de construção em painéis MDP.

Dessa forma, diantedas informações apresentadas, esta pesquisa objetivou colaborar com a literatura ao analisar o comportamento dos painéis particulados de madeira tratada com preservativo CCA, resina poliuretana à base de óleo de mamona e com adição de cimento em substituição às partículas de madeira, e comparando-o com os requisitos da norma brasileira NBR 14810 (ABNT, 2013).

\section{Materiais e métodos}

A fabricação e a caracterização dos painéis MDP da presente pesquisa foram desenvolvidas nas dependências do Laboratório de Estruturas de Madeira (LaMEM) do Departamento de Engenharia de Estruturas (SET) da Escola de Engenharia de São Carlos, da Universidade de São Carlos (USP). Foram utilizadas partículas de madeira da espécie Pinus sp. com granulometria até 2,8 mm e densidade aparente igual a $700 \mathrm{~kg} / \mathrm{m}^{3}$, obtidas no gerador de partículas localizado no laboratório (NASCIMENTO et al., 2015). Além disso, as partículas apresentavam teor de umidade próximo de $12 \%$, seguindo prescrições da norma brasileira NBR 7190 (ABNT, 1997), além de serem tratadas com CCA. O teor de adesivo em relação à massa seca das partículas é apresentado na Tabela 1. A resina utilizada foi a resina poliuretana bicomponente à base de óleo de mamona. Para a resina, adotou-se a proporção de 1:1 de poliol e prépolímero.

$\mathrm{O}$ cimento Portland (CP-V ARI) foi adicionado à mistura de partículas de madeira e resina nas proporções de $0 \%, 10 \%, 20 \%$ e $30 \%$ da massa de partículas, sendo produzidos 4 painéis para cada proporção (tratamento), totalizando 16 painéis. Cabe destacar que as frações mássicas de cimento irão substituir as de madeira, conferindo painéis com densidades nominais independentes dos tratamentos delineados, ilustrado na Tabela 1, e que a quantidade de adesivo se manteve constante em relação ao utilizado nas chapas de $0 \%$ de cimento (utilizadas como referência). Na confecção dos painéis não ocorre a adição de água, não havendo, assim, processo de hidratação do cimento, com este tendo função de preencher vazios.

Deu-se especial atenção ao espalhamento do cimento com as partículas, utilizando-se de espalhamento manual seguido de $5 \mathrm{~min}$ em batedeira elétrica e, por fim, de outro espalhamento manual. Os painéis foram compactados em uma prensa hidráulica, à temperatura de $100{ }^{\circ} \mathrm{C}$, pressão igual a $4 \mathrm{MPa}$ e tempo de prensagem de $10 \mathrm{~min}$. As dimensões dessas placas foram de $400 \mathrm{~mm} \times 400 \mathrm{~mm}$, com espessura de $10 \mathrm{~mm}$ (NASCIMENTO et al., 2015).

As placas foram estabilizadas e sofreram cura completa durante $72 \mathrm{~h}$, sendo posteriormente esquadrejadas nas dimensões de $380 \mathrm{~mm} \times 380 \mathrm{~mm}$ a fim de se obterem os corpos de prova para a realização dos testes físicos e mecânicos em temperatura ambiente, no LaMEM.

Foram confeccionados 3 corpos de prova (CP) de dimensões $50 \mathrm{~mm}$ x $50 \mathrm{~mm}$ com $10 \mathrm{~mm}$ de espessura para cada painel, totalizando $48 \mathrm{CP}$ para determinação das propriedades físicas de densidade aparente $(\rho)$, absorção de água após 2 h (Ab-2h) e após 24 h (Ab-24h) de imersão, e o inchamento em espessura após 2 h (Ic-2h) e após 24 h (Ic-24h) de imersão, conforme as premissas da norma brasileira NBR 14810 (ABNT, 2013).

Para a avaliação das propriedades mecânicas, foram preparados 3 corpos de prova nas dimensões de $250 \mathrm{~mm}$ x $50 \mathrm{~mm}$ (Figura 1) para a obtenção do módulo de elasticidade (MOE) e do módulo de ruptura (MOR), seguindo também as especificações da NBR14810 (ABNT, 2013).

Tabela 1 - Composição dos painéis produzidos

\begin{tabular}{c|c|c}
\hline Cimento (\%) & Partículas (g) & Adesivo (\%) \\
\hline 0 & 1.323 & 10 \\
10 & 1.190 & 10 \\
15 & 1.058 & 10 \\
20 & 926 & 10 \\
\hline
\end{tabular}


Figura 1 - Corpos de prova para avaliação das propriedades mecânicas

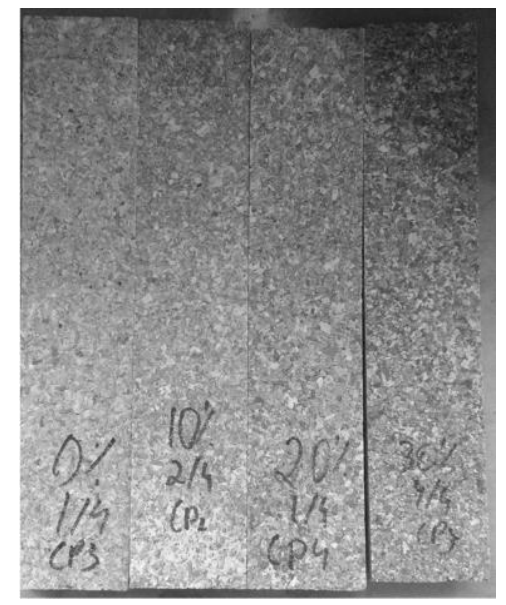

Para avaliar a influência da inclusão das frações de cimento nas propriedades físicas e mecânicas dos painéis, foi utilizada a análise de variância (ANOVA). A ANOVA foi considerada ao nível de 5\% de significância $(\alpha)$, com a equivalência das médias como hipótese nula $\left(\mathrm{H}_{0}\right)$ e a não equivalência de pelo menos duas como hipótese alternativa $\left(\mathrm{H}_{1}\right)$. Pela formulação das hipóteses, P-valor superior a 0,05 implica aceitar $\mathrm{H}_{0}$, e abaixo, refutá-la. Para validação da ANOVA foi utilizado o teste de comparações múltiplas de Tukey (teste de contraste de médias), de forma a realizar o agrupamento dos níveis do fator para cada tratamento nos diversos ensaios.

\section{Resultados e discussão}

A Figura 2 ilustra os valores médios, os intervalos de confiança da média (ao nível de 95\% de confiabilidade), os valores extremos do coeficiente de variação (CV) e os resultados do Teste de Tukey (5\% de significância) das propriedades densidade $(\rho)$, módulo de elasticidade (MOE) e módulo de resistência (MOR) na flexão estática referente aos quatro tratamentos experimentais delineados.

Os valores de densidade, MOE e MOR para os painéis de referência ( $\% \%$ cimento) são próximos daqueles obtidos por Bertolini et al. (2014a). Do Teste de Tukey, a $0 \%$ ou $10 \%$ de cimento forneceram os menores valores da densidade; $20 \%$ resultou no segundo maior valor; e $30 \%$ fornece o maior valor dessa propriedade. Isso se justifica pela adição de frações mássicas de cimento, que possuem densidade nominal de $3,5 \mathrm{~g} / \mathrm{cm}^{3}$, segundo o fabricante.

Para o MOE, $0 \%$ ou $10 \%$ de cimento forneceram os maiores valores médios dessa propriedade; $20 \%$ resultou no segundo maior valor médio; e 30\% de cimento impactou no menor valor dessa propriedade. É possível supor que essa redução do módulo de elasticidade seja devido ao aumento da área superficial das partículas que compõem o painel, proporcionando diminuição na adesão interna, já que a quantidade de adesivo se manteve constante. Além disso, é notável o aumento do coeficiente de variação nos resultados de MOE e MOR, o que corrobora a observação visual de que os painéis com maior concentração de cimento possuem pequenas concentrações de cimento em que o adesivo não se espalha bem, causando maior variabilidade nas propriedades mecânicas da chapa. Portanto, os únicos painéis que atendem aos requisitos para valor de MOE da norma NBR 14810 (ABNT, 2013) são os de tratamento igual a 0\% e 10\%.

Os maiores valores do MOR foram provenientes de $0 \%$ ou $10 \%$ de cimento, enquanto os menores foram oriundos de $20 \%$ ou $30 \%$ de cimento. Analogamente, observou-se a presença de pequenas aglomerações de cimento, aumentando a variabilidade dos valores obtidos e reduzindo o módulo de ruptura dos painéis com maior porcentagem de cimento. Novamente, os únicos painéis que atendem com segurança aos requisitos da norma brasileira para valores de MOR são os de tratamento igual a $0 \%$ e $10 \%$.

A Figura 3 ilustra os valores médios, os intervalos de confiança da média (ao nível de 95\% de confiabilidade), os valores extremos do coeficiente de variação (CV) e os resultados do Teste de Tukey (5\% de significância) das propriedades de absorção de água após 2 h (Ab-2h) e após 24 h (Ab-24h) de imersão, e o inchamento em espessura após 2 h (Ic-2h) e após 24 h (Ic-24h) de imersão.

664 Couto, J. P. M.; Nascimento, M. F.; Aquino, V. B. de M.; Panzera, T. H.; Christoforo, A. L.; Lahr, F. A. R. 
Figura 2- Resultados das propriedades avaliadas: densidade (a), módulo de elasticidade (b), módulo de resistência na flexão estática (c)

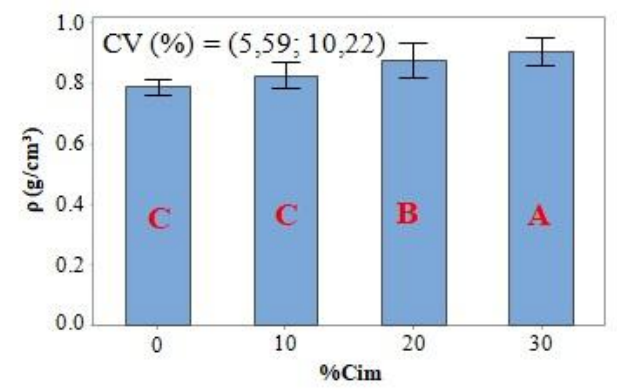

(a)

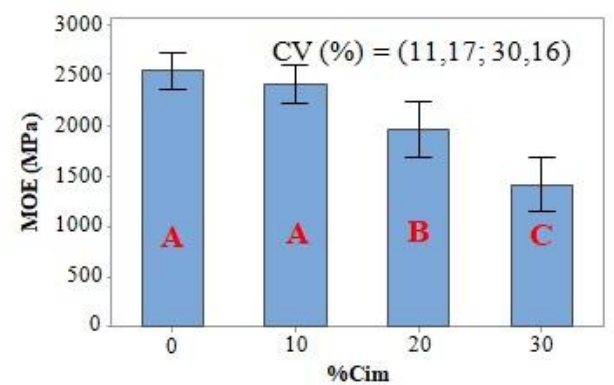

(b)

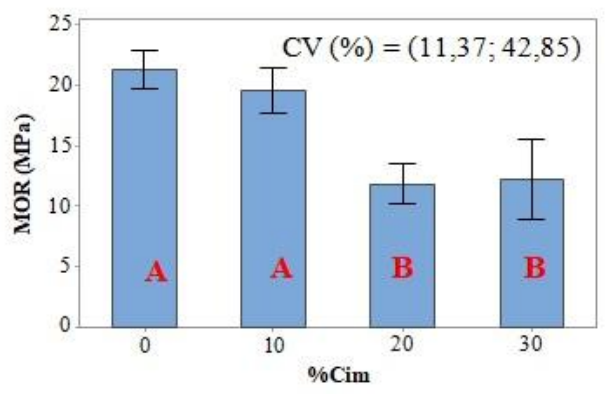

(c)

Figura 3- Resultados das propriedades físicas avaliadas: absorção de água após 2 h(a) e 24 h (b) de imersão, e inchamento em espessura após $2 \mathrm{~h}$ (c) e $24 \mathrm{~h}$ (d)

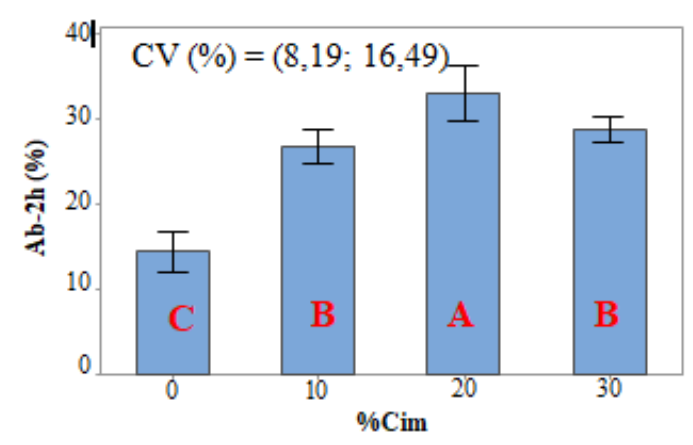

(a)

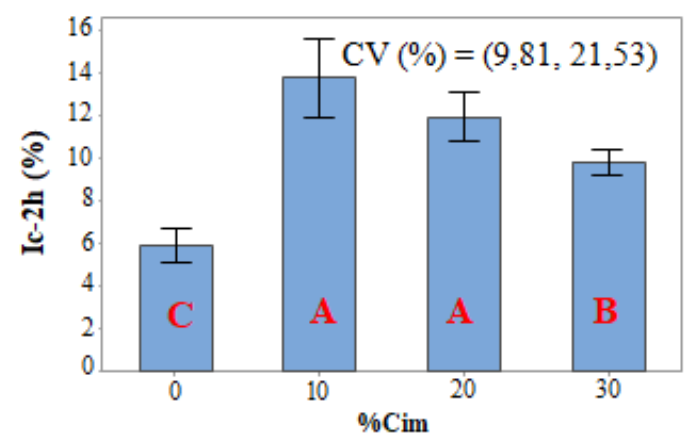

(c)

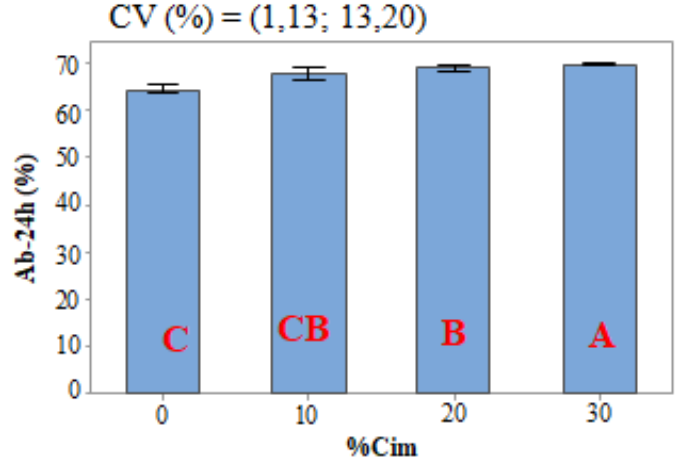

(b)

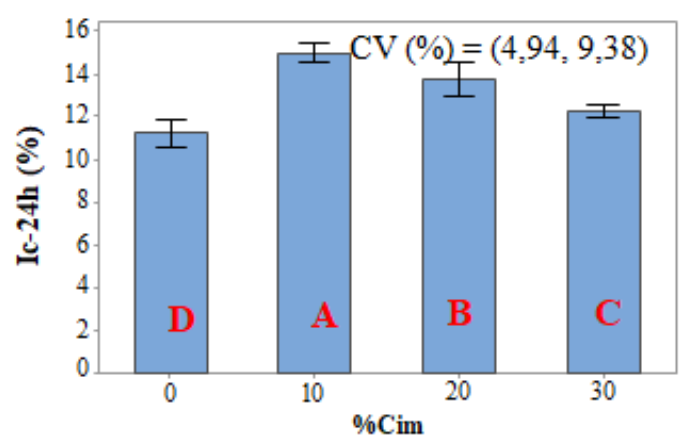

(d) 
Os valores de $\mathrm{Ab}-2 \mathrm{~h}, \mathrm{Ab}-24 \mathrm{~h}$, Ic-2h e Ic-24h para os painéis de referência ( $0 \%$ cimento) são próximos daqueles obtidos por Bertolini et al. (2014a). Do Teste de Tukey, a proporção0\% de cimento forneceu os menores valores de $\mathrm{Ab}-2 \mathrm{~h} ; 10 \%$ e $30 \%$ resultaram no segundo maior valor médio; e $20 \%$ forneceu os maiores valores de $\mathrm{Ab}-2 \mathrm{~h}$. Analogamente, essa configuração é similar para $\mathrm{Ab}-24 \mathrm{~h}$, mas com valores médios mais próximos, o que indica pouca influência das inclusões de cimento na absorção de água em mais de 24 h. Pelos resultados obtidos, todos os tratamentos atenderam às especificações da NBR 14810 (ABNT, 2013) para absorção de água.

Já para os ensaios de inchamento de espessura, do Teste de Tukey, 0\% de cimento forneceu os menores valores médios; $10 \%$ e $20 \%$ de cimento resultaram no maior valor; e $30 \%$ forneceu valores intermediários do Ic-2h. Analogamente, essa configuração se assemelha para o Ic-24h, mas com aumento significativo do inchamento nos painéis com tratamentos de $10 \%$ ou mais de cimento. Ainda assim, todos os tratamentos atenderam às especificações da NBR 14810 (ABNT, 2013) para inchamento após submersão em água.

\section{Conclusões}

Os resultados da presente pesquisa possibilitam concluir que:

(a) a adição de frações mássicas de cimento acima de $10 \%$ diminui significantemente os valores do módulo de elasticidade (MOE) e do módulo de ruptura (MOR) dos painéis de média densidade na flexão estática;

(b) a absorção de água após imersão por $2 \mathrm{~h}(\mathrm{Ab}-2 \mathrm{~h})$ de painéis tratados com cimento é maior que a de painéis com $0 \%$, entretanto a absorção após imersão por $24 \mathrm{~h}$ (Ab-24h) é muito próxima para todos os tratamentos; e

(c) em geral, os painéis com 10\%, 20\% e 30\% de cimento apresentam maior inchamento na espessura do que os painéis com $0 \%$ tanto após 2 h (Ic-2h) quanto após 24 h (Ic-24h) de imersão, mas ainda respeitam as especificações da Norma Brasileira NBR 14810 (ABNT, 2013) nesse quesito. Tais resultados enriquem a literatura sobre o tema, indicando que, apesar de a adição de materiais granulares como o cimento diminuir as propriedades mecânicas dos painéis de madeira, as outras propriedades físicas continuam a respeitar as determinações da norma vigente. Dessa forma, pesquisas mais abrangentes podem buscar maneiras de solucionar os problemas de acúmulo de materiais agregados nas chapas e de garantir melhor espalhamento e concentrações de adesivo para painéis tratados com cimento ou resíduos de construção civil.

\section{Referências}

ALMEIDA, A. S. et al. Influence of CCA-A preservative on physical-mechanical properties of Brazilian tropical woods. BioResources, v. 14, n. 2, p. 3030-3041, 2019.

AMIANDAMHEN, S. O.; MEINCKEN, M.; TYHODA, L. Magnesium based phosphate cement binder for composite panels: a response surface methodology for optimisation of processing variables in boards produced from agricultural and wood processing industrial residues. Industrial Crops and Products, v. 94, p. 746-754, 2016.

ARAUJO, V. A. et al. Difficulties of wooden housing production sector in Brazil. Wood Material Science and Engineering, p. 1-10, 2018.

ASSOCIAÇÃO BRASILEIRA DE NORMAS TÉCNICAS. NBR 14810: chapas de madeira aglomerada. Rio de Janeiro, 2013.

ASSOCIAÇÃO BRASILEIRA DE NORMAS TÉCNICAS. NBR 7190: projeto de estruturas de madeira. Rio de Janeiro, 1997.

BEECH, E. et al. Global Tree Search: the first complete global database of tree species and country distributions. Journal of Sustainable Forestry, v. 36, n. 5, p. 454-489, 2017.

BERTOLINI, M. S. et al.Painéis de partículas provenientes de rejeitos de Pinus sp. tratado com preservante CCA e resina derivada de biomassa. Revista Árvore, v. 38, n. 2, p. 339-346, 2014b.

BERTOLINI, M. S. et al. Particulate composites with wastes from treated wood and tire rubber. Advanced Materials Research, v. 1025-1026, p. 288-291, 2014a.

CARVALHO, A. G. et al. Use of tannin adhesive from Stryphnodendron adstringens (Mart.) Coville in the production of OSB panels. European Journal of Wood and Wood Products, v. 72, n. 4, p. 425-432, 2014.

666 Couto, J. P. M.; Nascimento, M. F.; Aquino, V. B. de M.; Panzera, T. H.; Christoforo, A. L.; Lahr, F. A. R. 
CHOI, C. et al. Analysis of mechanical properties of cross-laminated timber (CLT) with plywood using Korean larch. BioResources, v. 13, n. 2, p. 2715-2726, 2018.

DERKOWSKI, A.; MIRSKI, R.; MAJKA, J. Determination of sorption isotherms of scots pine (Pinus sylvestris L.) Wood strands loaded with melamine-urea-phenol-formaldehyde (Mupf) resin. Wood Research, v. 60, n. 2, p. 201-210, 2015.

FERRO, F. S. et al. Painel híbrido OSB/MDP de madeira Pinus taeda e resina poliuretana à base de óleo de mamona. Ambiente Construído, Porto Alegre, v. 19, n. 3, p. 7-14, jul./set. 2019.

FERRO, F. S. et al. Physical properties of OSB panels manufactured with CCA and CCB treated schizolobium amazonicum and bonded with castor oil-based polyurethane resin. International Journal of Materials Engineering,v. 6, n. 5, p. 151-154, 2016.

GARZÓN-BARRERO, N. M. et al. Evaluation of mold growth on sugarcane bagasse particleboards in natural exposure and in accelerated test. International Biodeterioration and Biodegradation, v. 115, p. 266-276, 2016.

GONÇALEZ, J. C. et al. Características tecnológicas das madeiras de Eucalyptus grandis W.Hill ex Maiden e Eucalyptus cloeziana F. Muell visando ao seu aproveitamento na indústria moveleira. Ciencia Florestal, v. 16, p. 329-341, 2006.

IHNÁT, V. et al. Waste agglomerated wood materials as a secondary raw material for chipboards and fibreboards part II: preparation and characterization of wood chips in terms of their reuse. Wood Research, v. 62 , n. 1, p. 45-56, 2017.

INDÚSTRIA BRASILEIRA DE ÁRVORES. Relatório 2017. São Paulo, 2017.

IWAKIRI, S. et al. Production of High Density particleboard using melamine-urea-formaldehyde resin. Cerne, v. 11, n. 4, p. 323-328, 2015.

LI, M.; SHI, J. Review on micropore grade inorganic porous medium based form stable composite phase change materials: preparation, performance improvement and effects on the properties of cement mortar. Construction and Building Materials, v. 194, p. 287-310, 2019.

MACEDO, L. B. et al. Propriedades físicas de painéis aglomerados de madeira produzidos com adição de película de polipropileno biorientado. Revista Brasileira de Engenharia Agrícola e Ambiental, v. 19, n. 7, p. 674-679, 2015.

MANTANIS, G. I. et al. Adhesive systems used in the European particleboard, MDF and OSB industries. Wood Material Science and Engineering, v. 13, n. 2, p. 104-116, 2018.

MISSAGIA, Z. M. V. et al. Materiais compósitos particulados em matriz epóxi reforçados com serragem, cimento e silicato de magnésio. Ambiente Construído, Porto Alegre, v. 13, n. 3, p. 285-302, jul./set. 2013.

MOLINA, J. C.; CALIL JUNIOR, C. Sistema construtivo em "wood frame" para casas de madeira.

Semina:Ciências Exatas e Tecnológicas, v. 31, n. 2, p. 143, 2010.

MONTGOMERY, D. G. Workability and compressive strength properties of concrete containing recycled concrete aggregate. Sustainable Construction: Use of Recycled Concrete Aggregate, p. 287-296, 1998.

NASCIMENTO, M. F. et al. Painéis OSB fabricados com madeiras da caatinga do nordeste do Brasil. Ambiente Construído, Porto Alegre, v. 15, n. 1, p. 41-48, jan./mar. 2015.

NEGRÃO, W. H. et al. Painéis aglomerados fabricados com mistura de partículas de madeiras tropicais. Ambiente Construído, Porto Alegre, v. 14, n. 3, p. 103-112, jul./set. 2014.

OLIVEIRA, S. L. et al. The effect of post-heat treatment in MDF panels. Materials Research, v. 20, n. 1, p. 183-190, 2017.

PANZERA, T. H. et al. Propriedades mecânicas de materiais compósitos à base de cimento Portland e resina epoxi. Cerâmica, v. 56, n. 337, p. 77-82, 2010.

RAMAGE, M. H. et al. The wood from the trees: the use of timber in construction. Renewable and Sustainable Energy Reviews, v. 68, p. 333-359, 2017.

ROFFAEL, E.; SCHNEIDER, T.; DIX, B. Influence of moisture content on the formaldehyde release of particle- and fiberboards bonded with tannin-formaldehyde resins. European Journal of Wood and Wood Products, v. 73, n. 5, p. 597-605, 2015. 
SCATOLINO, M. V. et al. Eucalyptus wood and coffee parchment for particleboard production: physical and mechanical properties. Ciência e Agrotecnologia, v. 41, n. 2, p. 139-146, 2017.

SEMPLE, K. E.; ZHANG, P. K.; SMITH, G. D. Hybrid oriented strand boards made from Moso bamboo (Phyllostachys pubescens Mazel) and Aspen (Populus tremuloides Michx.): species-separated three-layer boards. European Journal of Wood and Wood Products, v. 73, n. 4, p. 527-536, 2015.

SILVA, D. W. et al. MDP com partículas de eucalipto e palha de milho. Scientia Forestalis, v. 43, n. 108, p. 853-862, 2015.

SILVA, J. V. F. et al. Characterization of particleboards produced with Pinus spp. waste. Scientia Forestalis, v. 44, n. 111, p. 623-628, 2016.

SILVA, M. R. et al. Hybrid sandwich particleboard made with sugarcane, Pinus taeda thermally treated and malva fibre from Amazon. Materials Research, v. 21, n. 1, p. 7, 2018.

SILVA, S. A. M. et al. Medium density particleboard (MDF) produced with Pinus caribaea fibers and castor oil based polyurethane resin. Advanced Materials Research, v. 1025-1026, p. 13-16, 2014.

SOTSEK, N. C.; SANTOS, A. P. L. Panorama do sistema construtivo light wood frame no Brasil. Ambiente Construído, Porto Alegre, v. 18, n. 3, p. 309-326, jul./set. 2018.

SOUZA, A. M. et al. Mechanical properties of OSB wood composites with resin derived from a renewable natural resource. International Journal of Composite Materials, v. 4, n. 3, p. 157-161, 2014.

SOUZA, A. M. et al. Wood-based composite made of wood waste and epoxy based ink-waste as adhesive: a cleaner production alternative. Journal of Cleaner Production, v. 193, p. 549-562, 2018.

STEEGE, H. T. et al. The discovery of the Amazonian tree flora with an updated checklist of all known tree taxa. Scientific Reports, v. 6, p. 1-15, 2016.

THOMPSON, R. J. H. et al. Fatigue in wood-based panels. Part 1: The strength variability and fatigue performance of OSB, chipboard and MDF. Wood Science and Technology, v. 36, n. 3, p. 255-269, 2002.

VALARELLI, I. D. et al. Evaluation of bamboo particleboards produced with urea-formaldheyde resin. Advanced Materials Research, v. 1025-1026, p. 432-435, 2014.

ZAU, M. D. L. et al. Avaliação das propriedades química, física e mecânica de painéis aglomerados produzidos com resíduo de madeira da Amazônia - Cumaru (Dipteryx odorata) e resina poliuretana à base de óleo de mamona. Polímeros, v. 24, n. 6, p. 726-732, 2014.

\section{Agradecimentos}

Os autores agradecem à Coordenação de Aperfeiçoamento de Pessoal de Nível Superior (Capes) e ao Conselho Nacional de Desenvolvimento Científico e Tecnológico (CNPq) pelo apoio no desenvolvimento das pesquisas realizadas. 


\begin{abstract}
João Paulo Monteiro Couto
Departamento de Engenharia Civil | Universidade Federal de São Carlos | Rodovia Washington Luís, km 235, SP-310 | São Carlos - SP Brasil | CEP 36307-352 | Tel.: (16) 3351-8262 | E-mail: jpmcouto23@gmail.com
\end{abstract}

\title{
Maria Fátima Nascimento
}

Escola de Engenharia de São Carlos | Universidade de São Paulo | Av. Trabalhador Saocarlense, 400, Parque Arnold Schimit | São Carlos SP - Brasil | CEP 13566-590 | Tel.: (16) 3373-9369 | E-mail: fatinasc357@alumni.usp.br

Vinícius Borges de Moura Aquino

Instituto de Engenharia do Araguaia | Universidade Federal do Sul e Sudeste do Pará | Rua Geraldo Ramalho, s/n | Santana do Araguaia PA- Brasil | CEP 68560-000 | Tel.: (94) 2101-5936 | E-mail: aquino.vini@hotmail.com

\section{Tulio Hallak Panzera}

Departamento deEngenharia Mecânica | Universidade Federal de São João Del-Rei | Praça Frei Orlando, 170, Campus Santo Antônio | São João Del Rei - MG - Brasil | CEP 36300-000 | Tel.: (32) 3379-2395 | E-mail: panzera@ufsj.edu.br

\section{André Luis Christoforo}

Departamento de Engenharia Civil | Universidade Federal de São Carlos | E-mail: christoforoal@yahoo.com.br

\section{Francisco Antonio Rocco Lahr}

Escola de Engenharia de São Carlos | Universidade de São Paulo | Tel.: (16) 3373-9369 | E-mail: frocco@sc.usp.br

\section{Ambiente Construído}

Revista da Associação Nacional de Tecnologia do Ambiente Construído

Av. Osvaldo Aranha, $99-3^{\circ}$ andar, Centro

Porto Alegre - RS - Brasil

CEP $90035-190$

Telefone: +55 (51) 3308-4084

Fax: +55 (51) 3308-4054

www.seer.ufrgs.br/ambienteconstruido

E-mail: ambienteconstruido@ufrgs.br

This is an open-access article distributed under the terms of the Creative Commons Attribution License. 